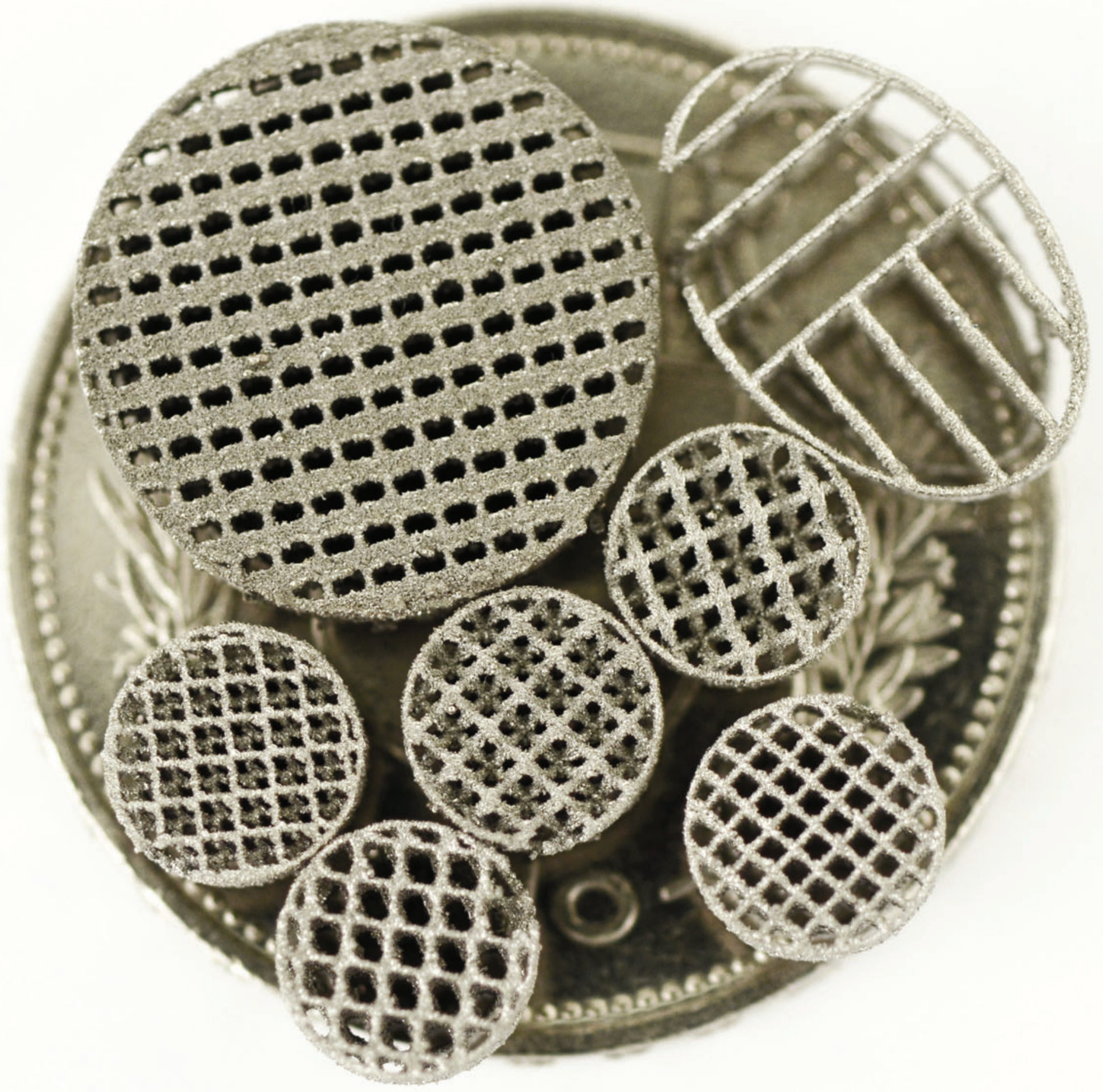




\title{
Influence of Microarchitecture on Osteoconduction and Mechanics of Porous Titanium Scaffolds Generated by Selective Laser Melting
}

\author{
Michael de Wild,' Simon Zimmermann, Jasmine Rüegg,, Ralf Schumacher, Thea Fleischmann,2 \\ Chafik Ghayor, and Franz E. Weber ${ }^{3}$
}

\begin{abstract}
Bone regeneration is naturally based on bone forming cells, osteoinduction by diverse growth factors, and osteoconduction. The latter one used as term in this study is the ingrowth of bone in 3D structures, which leads to an optimal case in creeping substitution of the scaffold by newly formed bone. Autologous bone is still the gold standard for bone substitutes. In most cases, newly developed bone substitutes consist of calcium phosphate, since hydroxyapatite is the main component of bone and mimics cancellous bone in microstructure. In this study, we wanted to elucidate the optimal microarchitecture for osteoconduction and determine compression strength and Young's Modulus of the selected architectures. Selective laser melting of titanium was used as tool to generate diverse architectures in a repetitive and precise way. To link 3D scaffold architecture to biological readouts, bone ingrowth, bone to implant contact, and defect bridging of noncritical-sized defects in the calvarial bone of rabbits were determined. In this series, 5 different microarchitectures were tested with pore sizes ranging from 700 to $1300 \mu \mathrm{m}$ and constrictions between 290 and $700 \mu \mathrm{m}$. To our surprise, all microstructures showed the same biological response of excellent osteoconduction. However, the mechanical yield strength of these structures differed by the factor of three and reached up to three times the strength of cancellous bone at a porosity of 72.3-88.4\%. These results suggest that the microarchitecture of bone substitutes can be optimized toward mechanical strength in a wide range of constrictions and pore sizes without having a negative influence on osteoconduction.
\end{abstract}

Keywords: bone substitute, microarchitecture, open-porous, osteoconduction, scaffolds, titanium

\section{Introduction}

Personalized MEDicine is based on a fast evaluation of the patient's needs and the application of a patient-specific treatment. For the treatment of large bony defects, which pose still a challenge in orthopedic and craniomaxillofacial surgery, it translates mainly into the determination of the overall shape of the defect and the production of a patient-specific bone substitute of the shape determined beforehand.

From the biological point of view, bone regeneration and healing of bony defects are facilitated by osteoconduction, osteoinduction, and the transplantation of bone cells. The latter one is normally realized by transplantation of autografts, where bone from a healthy site is transplanted to the diseased site $^{1}$ or in rare occasions by the use of bone marrow

\footnotetext{
${ }^{1}$ School of Life Sciences, Institute for Medical and Analytical Technologies, University of Applied Sciences Northwestern Switzerland, Muttenz, Switzerland.

${ }^{2}$ Division of Surgical Research, Centre for Clinical Research University Hospital Zurich, Switzerland.

${ }^{3}$ Oral Biotechnology \& Bioengineering, Center of Dental Medicine, University of Zurich, Zurich, Switzerland.
}

Opposite page: Osteoconductive titanium based bone substitute variations realized by laser sintering. The samples are arranged on a 5 Swiss Franc coin (Diameter $31.45 \mathrm{~mm}$ ).

(C) Michael de Wild, et al., 2016; Published by Mary Ann Liebert, Inc. This Open Access article is distributed under the terms of the Creative Commons Attribution Noncommercial License (http://creativecommons.org/licenses/by-nc/4.0/) which permits any noncommercial use, distribution, and reproduction in any medium, provided the original author(s) and the source are credited. 
stem cells harvested from the iliac crest. ${ }^{2,3}$ Osteoinduction as the potential to induce bone formation even at ectopic sites by cell recruitment and differentiation into preosteoblasts was discovered in the $1960 \mathrm{~s}^{4}$ and applied as bone morphogenetic protein-2 clinically from 2002 mainly for spinal fusion procedures. ${ }^{5}$ Osteoconduction describes bone regeneration by the potential of bone to grow onto an implant or into porous 3D structures and ideally leads to creeping substitution of the 3D structure by bone as already described in the late 1800s by Arthur Barth. " More recently the term "osteoconduction" was also used to describe bone growth on surfaces. ${ }^{7}$ Here we mainly refer to osteoconduction as bone growth into $3 \mathrm{D}$ structures.

The realization of a patient-specific bone substitute comprises its macroarchitecture as defined as the overall outer shape of the device, its inner microarchitecture reflecting the tissue morphology (e.g., pore size, shape, porosity, spatial distribution, channels, and pore interconnection), and its nanoarchitecture on the surface. The nanoarchitecture is either determined by the production methodology, for example, the grain size of the raw materials or the surface roughness of the fabricated scaffold, or by surface modifications applied after the initial production procedure (e.g., etching, sand blasting, spark anodization, sintering, or even biomolecule attachment for cell adhesion, proliferation, and differentiation). ${ }^{8,9}$

Additive manufacturing enables the realization of the macroarchitecture of the scaffold and allows the bone substitutes to perfectly fill a patient's bony defect. Maybe even more importantly, additive manufacturing facilitates the realization of a predefined microarchitecture throughout the scaffold needed for an optimal in vivo response in terms of enhanced bone regeneration by osteoconduction. Using powder metallurgy or chemical vapor deposition coating of vitreous carbon scaffolds, ${ }^{10,11}$ porous titanium scaffolds could only be produced with a homogeneous pore size distribution. Open porous titanium foams produced by metal injection molding with $\mathrm{NaCl}$ as placeholders result in a random distribution of the pores. ${ }^{12}$ Since all these methods fall short to predefine the exact microarchitecture in terms of location and size of micrometer-sized channels, pores, gradients, or patterns, we have chosen additive manufacturing by laser melting to study the relationship between microarchitecture and biological outcome, here defined as osteoconduction. Despite the limitations of former scaffolds with microarchitectures based on randomly distributed and therefore unsystematic and unplanned pores, those results indicate that pores with diameters between 150 and $500 \mu \mathrm{m}$ are best suited for bone substitute materials. ${ }^{13}$ More recently, pores of up to $1220 \mu \mathrm{m}$ showed also good results. ${ }^{14}$ Data on defined constrictions in scaffold microarchitecture and comparisons of a wide range of pore sizes for scaffolds made from one type of material are still elusive.

Recently, we determined osteoconduction as bone growth into 3D structures of titanium-based implants manufactured by additive manufacturing with defined macro- and microarchitecture but diverse in nanoarchitecture to fit a $6 \mathrm{~mm}$ noncritical-sized defect in the calvarial bone of rabbits. From all the implants produced by selective laser melting (SLM), we found the sand-blasted acid etched implants to be superior to native SLM and sand-blasted SLM ones. ${ }^{15}$ In this study here, we used the same model and tested native SLM implants of defined macro- and nanoarchitecture but different in microarchitecture types A-E for impact on osteoconduction and mechanical performance. The results showed that an optimization in mechanical functioning in the evaluated range of diverse microarchitectures could be achieved without compromising osteoconduction of the implants.

\section{Materials and Methods}

\section{Implant design}

The titanium-based scaffolds used in this study were designed with the Software Solidworks V.2013 (www .solidworks.com, Dassault Systèmes, France) and Magics

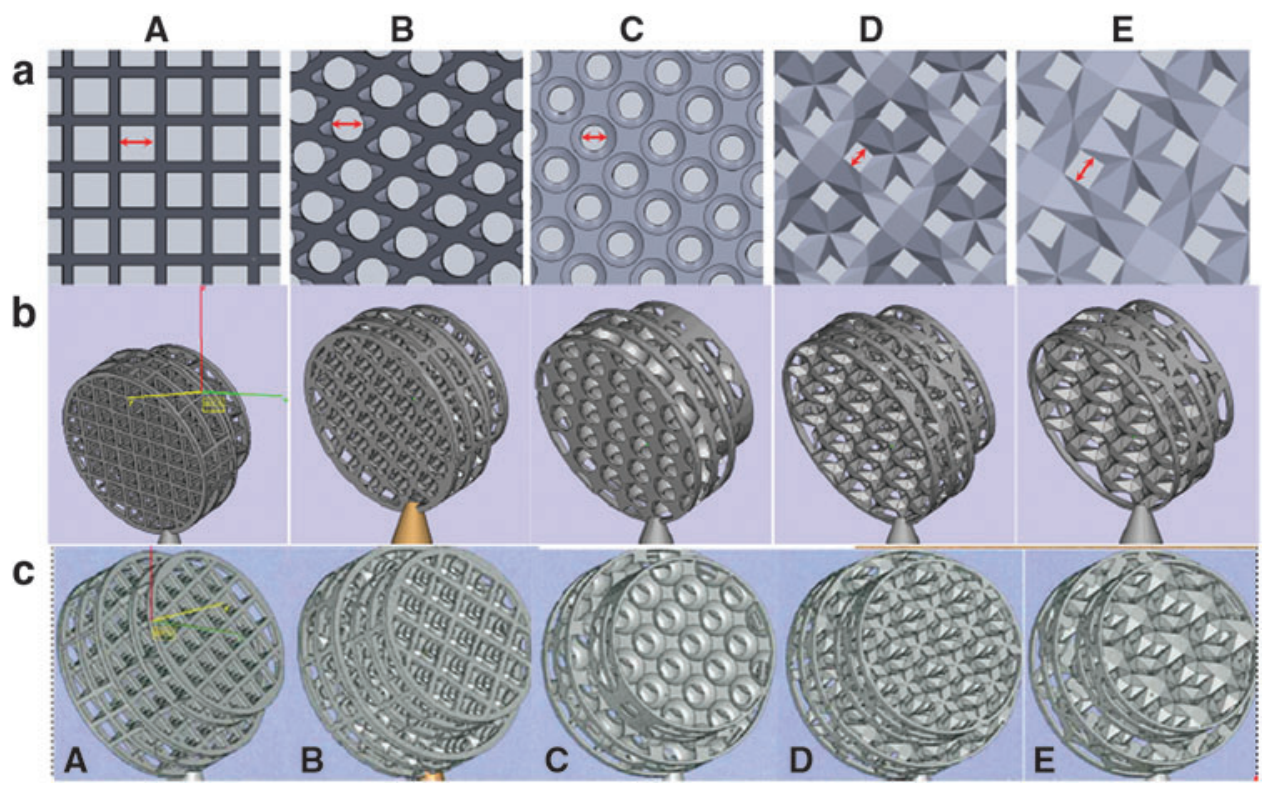

FIG. 1. Micro- and macroarchitecture of the scaffolds: (a) periodic 3D lattice of the microarchitecture types $A-E$ filling out and (b) stepped cylindrical macroshape of the scaffolds viewed from the backside and (c) from the front side. Main diameter of the macroscopic cylinder: $7.5 \mathrm{~mm}$, height: $3.8 \mathrm{~mm}$. Geometrical details of the microarchitecture are given in Table 1 . 
Table 1. Three-Dimensional Parameters of the Microarchitecture Types A-E

\begin{tabular}{|c|c|c|c|c|c|}
\hline $\begin{array}{l}\text { Type of } \\
\text { microarchitecture }\end{array}$ & $A$ & $B$ & $C$ & $D$ & $E$ \\
\hline Description of the unicell & $\begin{array}{l}\text { Three orthogonal } \\
\text { struts }\end{array}$ & $\begin{array}{l}\text { Cube with channels } \\
\text { along the room } \\
\text { diagonal }\end{array}$ & $\begin{array}{c}\text { Cube with a central } \\
\text { spherical cavity }\end{array}$ & $\begin{array}{l}\text { Dodecahedra } \\
\text { with } \\
\text { additional } \\
\text { struts to the } \\
\text { center of the } \\
\text { cell }\end{array}$ & $\begin{array}{l}\text { Dodecahedra with } \\
\text { additional } \\
\text { struts to the } \\
\text { center of the } \\
\text { cell }\end{array}$ \\
\hline Length unit cell (mm) & 0.90 & 1.10 & 1.20 & 1.41 & 1.79 \\
\hline $\begin{array}{l}\text { Size of microarchitectural } \\
\text { features }(\mathrm{mm})\end{array}$ & $\begin{array}{l}\text { Strut thickness } \\
0.20 \\
\text { Periodicity } 0.9\end{array}$ & $\begin{array}{l}\text { Diameter of } \\
\text { channels } 0.60\end{array}$ & $\begin{array}{l}\text { Diameter of central } \\
\text { cavity } 1.36\end{array}$ & $\begin{array}{l}\text { Strut } \\
\text { thickness } \\
0.40\end{array}$ & $\begin{array}{l}\text { Strut } \\
\text { thickness } \\
0.51\end{array}$ \\
\hline \multicolumn{6}{|l|}{ Unit cell } \\
\hline $\begin{array}{l}\text { Maximal constriction }(\mathrm{mm}) \\
\text { See arrows in Figure 1a }\end{array}$ & 0.70 & 0.6 & 0.66 & 0.29 & 0.38 \\
\hline Object volume $\left(\mathrm{mm}^{3}\right)$ & 8.39 & 12.65 & 19.99 & 17.77 & 18.00 \\
\hline Object volume $(\%)$ & 11.6 & 17.5 & 27.7 & 24.7 & 25.0 \\
\hline Open porosity (\%) & 88.4 & 82.5 & 72.3 & 75.3 & 75.0 \\
\hline Closed porosity (\%) & 0 & 0 & 0 & 0 & 0 \\
\hline Object surface $\left(\mathrm{mm}^{2}\right)$ & 184 & 243 & 181 & 258 & 206 \\
\hline Object-specific surface $\left(\mathrm{mm}^{-1}\right)$ & 21.9 & 19.2 & 9.2 & 14.5 & 11.5 \\
\hline Degree of anisotropy & 0.19 & 0.19 & 0.09 & 0.06 & 0.09 \\
\hline Connectivity & 176 & 326 & 60 & 135 & 96 \\
\hline
\end{tabular}

V.15 (www.materialise.com, Materialize NV, Leeuven, Belgium), based on unit cells as illustrated for the diverse building blocks (Fig. 1a and Table 1). All macroarchitectures consist of a stepped cylindrical shape with a total macroscopic height of $3.8 \mathrm{~mm}$ (Fig. 1b, c). The diameter of the larger macroscopic cylinder was $7.5 \mathrm{~mm}$, while the smaller outer diameter was $6 \mathrm{~mm}$. The microarchitectures are based on categorized open porous lattice types A-E.

The microarchitectures of the implants were constructed by replicating the corresponding unit cell types A-E as a space filling pattern in all spatial dimensions inside the staged cylinder (Fig. 1), leading to an overall porosity of $72.3-88.4 \%$. The lattice architecture type A consists of orthogonal struts with thickness $200 \mu \mathrm{m}$ and periodicity of $900 \mu \mathrm{m}$. The open channels (marked by arrow in Fig. 1a) have a dimension of $700 \mu \mathrm{m}$. Type B lattice is constructed by $600-\mu \mathrm{m}$-thick pores along all three room diagonals of the cubic unit cell with a unit cell length of $1.1 \mathrm{~mm}$. The lattice architecture type $\mathrm{C}$ is derived by repeatedly subtracting spheres of diameter $\varnothing 1.36 \mathrm{~mm}$ with a repetition distance of $1.2 \mathrm{~mm}$ in all three orthogonal directions, resulting in spherical cavities connected with each other by "bottle necks" of $660 \mu \mathrm{m}$ diameter. Lattice types $\mathrm{D}$ and $\mathrm{E}$ are both based on rhombohedral dodecahedron (RDH) symmetry, D with an elementary cell length of $1.41 \mathrm{~mm}$ and $E$ with $1.79 \mathrm{~mm}$. The strut size selected was $400 \mu \mathrm{m}$ for type D and $510 \mu \mathrm{m}$ for type E leading to final persistent channels with a diameter of $290 \mu \mathrm{m}$ and resp. $380 \mu \mathrm{m}$ for scaffold types D and E.

In addition, boundary rings are added around the lattice structure to define the border and to avoid sharp edges. A pin is added, which is necessary during the SLM building procedure, the post-treatment, and the surgical placement of the scaffold during implantation (Fig. 1b). It can easily be disconnected at the predetermined breaking point without destroying the scaffold.

\section{$3 D$ characterization}

Whereas simple geometrical parameters such as strut thickness and pore size from type A (Table 1) were derived directly from the geometrical definition, more complex 3D considerations were numerically calculated with the "3D analysisfunction" of the CTAn software (version 1.8.0.5; SkyScan $\mathrm{NV})^{16}$ from the stl-files of scaffold types A-E, excluding the symmetry breaking closing rings and the transfer pin. For all five implant types A-E, a cylindrical volume of interest (VOI) with a diameter of $5.08 \mathrm{~mm}$ and a height of $3.56 \mathrm{~mm}$ was selected to characterize the microarchitecture inside the scaffold resulting in a total VOI volume of $72.1 \mathrm{~mm}^{3}$.

\section{Implant production}

All implants were produced in the SLM Realizer $250^{\mathrm{HT}}$ Selective Laser Melting machine (SLM Solutions GmbH, Lübeck, Germany) operated with a continuous wave Ytterbium fiber laser with a wavelength between 1068 and $1095 \mathrm{~nm}$. Tipowder (Ti grade II according to Ref. ${ }^{17}$ with a $\mathrm{d}_{50}$-value of $60 \mu \mathrm{m}$ ) was used as raw material (SLM Solutions $\mathrm{GmbH}$ ). The powder layer thickness was set to $30 \mu \mathrm{m}$. The fusion of the titanium particles by the laser occurred at an energy density of $E_{v}$ of $63 \mathrm{~J} / \mathrm{mm}^{3}$. In analogy to Ref. ${ }^{15}$, the transfer pins were positioned vertically onto the building platform with the tilted scaffolds at the front end (Fig. 2a). After fabrication and excavation from the surrounding titanium powder, adhering powder particles were first removed by compressed air and then 


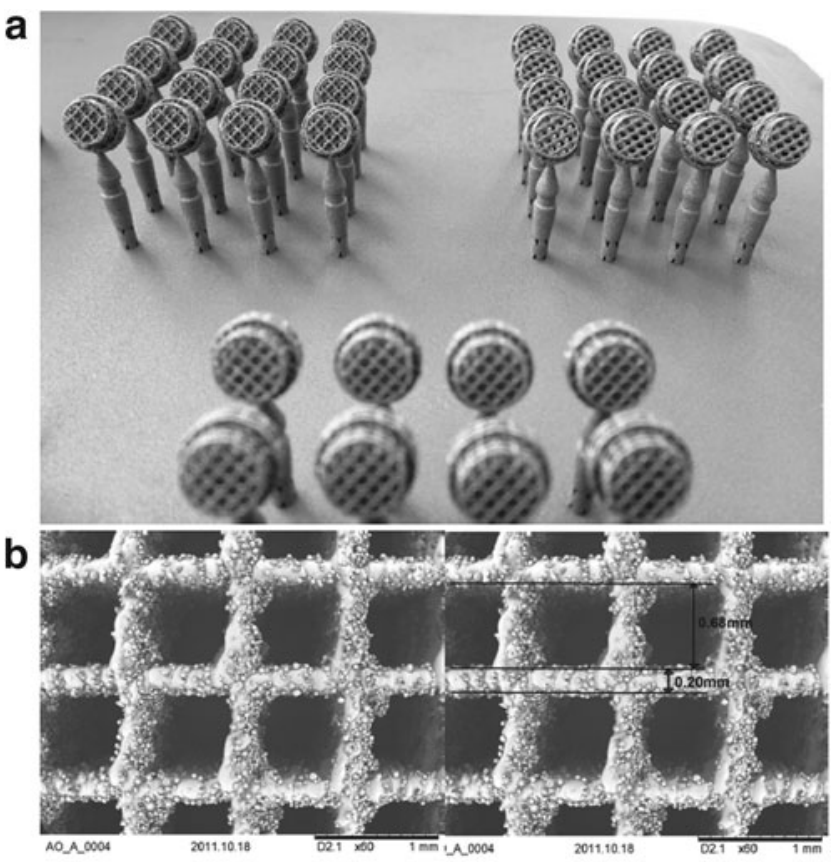

FIG. 2. Scaffolds on the selective laser melting (SLM) building platform after excavation and scanning electron microscopy: (a) Front: Type D, back left: Type E, back right: Type C. (b) Scanning electron microscopic image of the scaffold type A with native SLM surface. Scale bars indicate $1 \mathrm{~mm}$. The orthogonal lattice architecture with strut thickness of $200 \mu \mathrm{m}$ is decorated by residual titanium particles from the process.

by ultrasonic cleaning in a $4 \%$ Deconex ${ }^{\circledR} 15 \mathrm{PF}$ (Beiersdorf Münchenstein, Switzerland) solution at $90^{\circ} \mathrm{C}$ for $15 \mathrm{~min}$. Next, the implants were cleaned three times in ultrapure water (resistivity $18.2 \mathrm{M} \Omega \mathrm{cm}$ ) for $5 \mathrm{~min}$ in an ultrasonic bath with intermittent rinsing under flowing ultrapure water and thereafter were passivated two times in concentrated nitric acid $(32.5 \%$ $\mathrm{HNO}_{3}$, Fluka 84380) at room temperature under ultrasonic application for $10 \mathrm{~min}$. Finally, an ultrasonic cleaning and rinsing sequence in ultrapure water for $5 \mathrm{~min}$ was repeated three times. A final cleaning and sterilization procedure was done using RF plasma treatment with high-purity oxygen (PDC32G, Harrick, Ithaca, NY; oxygen purity $99.9995 \%$, Carbagas) at $29.7 \mathrm{~W}$ for $5 \mathrm{~min}$. No additional surface treatment was applied. After packaging and labeling, the implants were gamma sterilized with $25 \mathrm{kGy}$.

\section{Surgical procedure}

Eleven adult (12 months old) New Zealand White rabbits, weighing between 3.5 and $4.5 \mathrm{~kg}$, were used in the present study. The animals were housed in groups in a purposedesigned room for experimental animals and fed a standard laboratory diet. The methodology of animal trial was evaluated and accepted by the local authorities (108/2012). Animals were anesthetized by an injection of $65 \mathrm{mg} / \mathrm{kg}$ ketamine and $4 \mathrm{mg} / \mathrm{kg}$ xylazine and further anesthetized with isoflurane $/ \mathrm{O}_{2}$. The surgical area was disinfected and an incision was made from the nasal bone to the mid-sagittal crest. Next, the soft tissues were reflected and the periosteum was elevated from the site. In the area of the right and left parietal and frontal bones, four evenly distributed 6-mm-diameter craniotomy defects were prepared with a trephine bur under copious irrigation with sterile saline. For the completion of the defect, a rose burr $(1 \mathrm{~mm})$ was used to preserve the dura. Before implant placement, bone debris were removed by flushing with saline. Each of the animals received four different treatment modalities. In the first round, empty and design A implants were applied with two other implants, which are not included in this report. In the second round, all animals received implants of the designs B, C, D, and E. The treatment modalities were assigned at random for the first animal, and thereafter, cyclic permuted clockwise for the next three animals. For the fifth animal, treatment modalities were again assigned at random. After carefully placing the implants into the defects and detachment of the transfer pin, the soft tissues were closed with interrupted sutures. After a healing period of 8 weeks, the rabbits were placed under general anesthesia and sacrificed by an overdose of pentobarbital. The skull containing all four craniotomy sites was removed and placed in $40 \%$ ethanol.

\section{Embedding}

The specimens were prepared with a sequential water substitution process. It involved $48 \mathrm{~h}$ in $40 \%$ ethanol, $72 \mathrm{~h}$ in $70 \%$ ethanol (changed every $24 \mathrm{~h}$ ), $72 \mathrm{~h}$ in $96 \%$ ethanol, and finally, $72 \mathrm{~h}$ in $100 \%$ ethanol. Thereafter, samples were placed in xylene for $72 \mathrm{~h}$ (changed every $24 \mathrm{~h}$ ) followed by methyl methacrylate (MMA) for $72 \mathrm{~h}$ (Fluka 64200) and $100 \mathrm{~mL}$ $\mathrm{MMA}+2 \mathrm{~g}$ dibenzoylperoxid (Fluka 38581 ) at $4^{\circ} \mathrm{C}$ for 4 days. For polymerization, samples were submerged in $100 \mathrm{~mL}$ MMA $+3 \mathrm{~g}$ dibenzoylperoxid $+10 \mathrm{~mL}$ plastoid $\mathrm{N}$ or dibutylphthalate (Merck 80019.25 ) at $37^{\circ} \mathrm{C}$ in an incubator. After embedding, the skull was cut in four pieces each containing one craniotomy site by using an EXAKT 300P saw (Exakt, Norderstedt, Germany).

\section{Histomorphometry}

Histomorphometric analyses were performed from the middle sections using image analysis software (Image-Pro Plus $^{\mathrm{TM}}$; Media Cybernetic, Silver Springs, MD). The area of interest (AOI) was defined by the implant dimension. New bone formation in the AOI (new bone area, $\mathrm{mm}^{2}$ ), bone to implant contact (BIC, \%), and percent of bone in the AOI of the areas devoid of titanium (bone filling, \%) were determined. For the latter, empty control value of the average area occupied by titanium of all five designs was taken into account and subtracted from the AOI.

\section{Bone bridging}

Bone bridging was determined in the middle section. First, the areas with bone tissue were projected onto the $\mathrm{x}$-axis. Next, the stretches of the $\mathrm{x}$-axis where bone formation had occurred at any level were summed up as described earlier. ${ }^{18,19}$ Bone bridging is given in percentage of the defect width $(6 \mathrm{~mm})$ where bone formation has occurred.

\section{Evaluation of the mechanical properties of the implant material}

A universal material testing machine (Z100 THW allround-line, $100 \mathrm{kN}$ X-force load cell; Zwick GmbH \& Co. 
KG, Ulm, Germany) under uniaxial compressive load was used to analyze the mechanical properties of the porous scaffolds types A-E as well as solid SLM material. In agreement with the intra- and postoperative biomechanical loading, the implants were positioned between two hard metal compression inserts in a horizontal position. The samples were first preloaded with $2 \mathrm{MPa}$. Then, a constant strain of $0.001 \mathrm{~s}^{-1}$ was applied at room temperature, while the resulting force and deformation were recorded during the strain-controlled compression phase.

\section{Surface analysis}

To characterize the morphology of the nano- and microarchitecture, the implants and their surfaces were inspected by SEM (TM-1000, Hitachi, backscattered electrons, acceleration voltage $15 \mathrm{kV}$, energy dispersive $\mathrm{X}$-ray spectrometer EDX, SwiftED-TM detector). The surface roughness was quantified by a confocal laser scanning microscope (LEXT, Olympus OLS 3100 with a $50 \times$ objective) using a cutoff wavelength of $\lambda=1 / 10=10 \mu \mathrm{m}$.

\section{Statistical analysis}

The primary analysis unit was the animal. For all parameters tested, the 6 treatment modalities were compared with a Kruskal-Wallis test, followed by pairwise comparison of treatment modalities with the Mann-Whitney test for dependent data (IBM SPSS v.19). $p$-Values are displayed in the graphs and significance was set at $p<0.05$. Data from 11 rabbits are presented. Values are reported as mean \pm SD and displayed as box plots ranging from the 25th (lower quartile) to the 75th (upper quartile) percentile, including the median and whiskers showing the minimum and maximum.

\section{Results}

\section{Implant production}

The implants, including the transfer pins with the predetermined breaking point, were produced on the SLM building platform (Fig. 2a), whereas the bicylindrical macroarchitecture is identical for all scaffold types A-E, the five different microarchitectures are produced according to the designs described in Figure 1. The nanoarchitecture of the native SLM surface is shown in SEM images (Fig. 2b). The struts of, for example, scaffold type A have a thickness of $\sim 200 \mu \mathrm{m}$ and are decorated by residual titanium particles from the initial powder. The roughness, measured on the struts by confocal laser scanning microscopy, is $\mathrm{R}_{\mathrm{a}}=3.24 \pm 1.05 \mu \mathrm{m}, \mathrm{SR}_{\mathrm{a}}=3.37 \pm 0.27 \mu \mathrm{m}$, and $\mathrm{SR}_{\mathrm{z}}=46.88 \pm 4.23 \mu \mathrm{m}$. Due to this particular coverage, the actual surface is increased by an enlarging factor $\alpha=2.64$ compared to the projected area.

\section{$3 D$ characterization}

Basic geometrical parameters such as strut thickness, maximal constrictions, porosity, degree of anisotropy, object volume, object-specific surface, connectivity, and pore size are listed (Table 1). The porosity of the microarchitecture varies between $72.3 \%$ and $88.4 \%$. The diameter of the minimal constrictions lies between 0.29 and $0.7 \mathrm{~mm}$, showing that lattice type D features the tightest "bottle necks,"
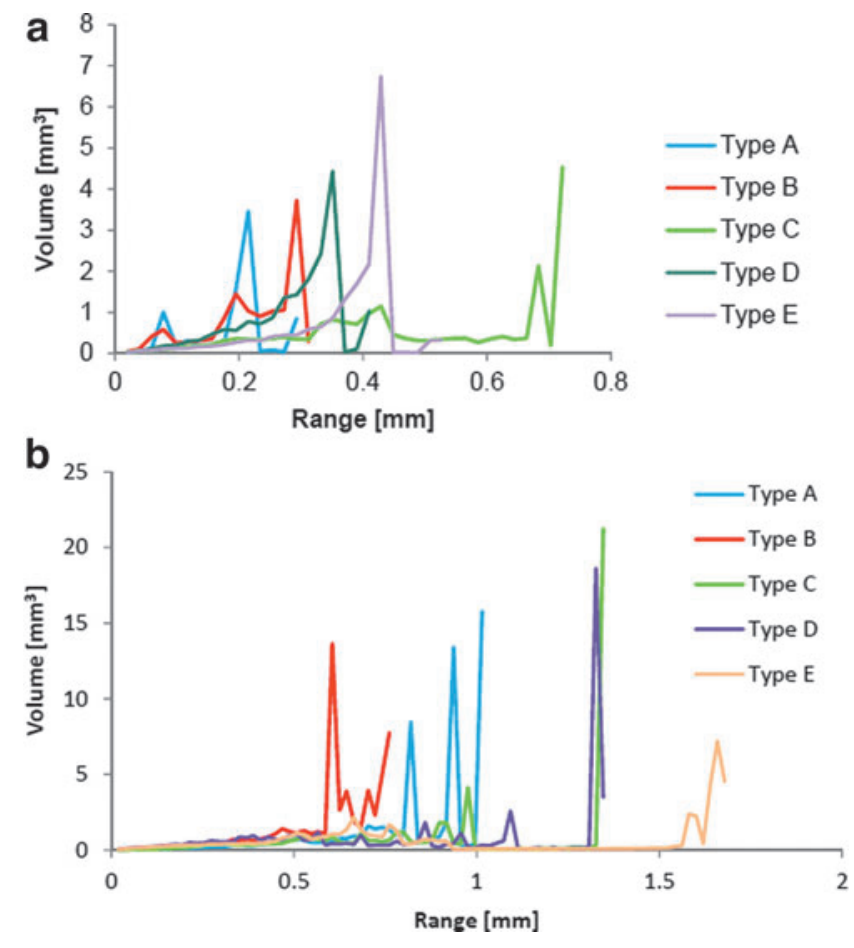

FIG. 3. Structure thickness distribution (a) and structure separation distribution (b) of microarchitectures $A-E$.

whereas all inner pores of lattice type A can be reached easily through a neck of $0.7 \mathrm{~mm}$ diameter.

Type A shows the most discrete structure thickness distribution since all struts have the same diameter throughout the scaffold (Fig. 3a). In the more complex designs such as in type B, C, D, and E with polygons, spheres, and radii, the thickness distribution shows broader and asymmetric peaks with shoulders.

The struts in type A microarchitecture have a thickness of $200 \mu \mathrm{m}$ and are separated by $0.7 \mathrm{~mm}$, characterized by the peak in the structure thickness distribution at $0.2 \mathrm{~mm}$ (Fig. 3a) and $\sim 0.8 \mathrm{~mm}$ in the structure separation distribution (Fig. 3b). The diagonal length of the elementary cell in type A is $0.99 \mathrm{~mm}$, which explains the second peak at $0.95 \mathrm{~mm}$ in the structure separation distribution (Fig. 3b). In type B lattices, the pillars between the channels have a diameter of $0.26 \mathrm{~mm}$, which is reflected in the first peak of the structure thickness distribution. The structure separation distribution of type B microarchitecture shows a peak at $\sim 0.6 \mathrm{~mm}$ (Fig. 3b), which corresponds to the channel diameter of $0.6 \mathrm{~mm}$. In cross points of the channels, the struts are separate by $0.73 \mathrm{~mm}$ leading to a second peak. The diameter of the empty sphere inside the elementary cell of type $\mathrm{C}$ is $1.36 \mathrm{~mm}$ and therefore explains the peak at $1.35 \mathrm{~mm}$ in the structure separation distribution (Fig. 3b), while the concave curvature leads to a broadened structure thickness distribution up to $0.45 \mathrm{~mm}$ (Fig. 3a). The peak at $0.7 \mathrm{~mm}$ corresponds to the massive corners remaining between the spheres with a thickness of $0.72 \mathrm{~mm}$. Both microarchitectures $\mathrm{D}$ and $\mathrm{E}$ are based on RDH cells, however, designed with different scales. Due to thicker struts in microarchitecture E compared with D, the asymmetric peak of the structure thickness distribution is shifted toward higher values 
(Fig. 3a). However, the aimed strut thickness of $0.4 \mathrm{~mm}$, resp. $0.5 \mathrm{~mm}$ are not well mapped, tails toward lower thicknesses appear due to the imprecise strut thickness in the digital stl-model (Table 1). The expected diameters of the $\mathrm{RDH}$ cavities are ca. $1.3 \mathrm{~mm}$ (type D) and ca. $1.6 \mathrm{~mm}$ (type E). The peaks in the structure separation distribution in type $\mathrm{D}$ are also shifted from 1.33 to $1.66 \mathrm{~mm}$ in lattice type $\mathrm{E}$ due to the upscaled elementary RDH cell (Fig. 3b).

In general, a smaller object volume correlates to a larger porosity of the same object (Table 1). Results for the objectspecific surface indicate that type A and B are the most complex/filigree microarchitectures, while type $\mathrm{C}$ is the least complex, that is, the most clumsy structure. This goes along with the results for connectivity, which states that microarchitectures type A and B are topologically more intensively connected than the other microarchitectures (i.e., more cuts are necessary for a geometrical separation). Furthermore, the degree of anisotropy of the microarchitectures type A and B is significantly larger compared to type $\mathrm{C}, \mathrm{D}$, and $\mathrm{E}$. This increased anisotropy reflects the enhanced preferential structural alignment along particular spatial axis of architectures $\mathrm{A}$ and $\mathrm{B}$.

\section{Mechanical characterization}

The values for the components Young's Modulus $E$ (with reference area of the small $\varnothing 6 \mathrm{~mm}$ cylinder cross section), the Yield Strength $R_{0.2}$, and the maximum strength before structural failure $R_{m}$ are listed (Table 2).

\section{Bone formation}

All treated animals showed an uneventful healing and stayed in good health. In the ground sections, no signs of inflammation could be detected. Bone formation occurred close to and in contact with the bone substitutes irrespective of the designs used (Fig. 4), which indicates a good biocompatibility over the first 8 weeks on implantation for all SLM implant designs.

The histomorphometric analysis (Fig. 5a) of the middle sections revealed that the area of newly formed bone of the designs $\mathrm{B}, \mathrm{C}$, and $\mathrm{D}$ was significantly higher $(p=0.08)$ than in the control group where no implant was placed. Between the groups with implants, the area of newly formed bone was not significantly different. The bone filling show a similar trend like the bone area (Fig. 5b). Over the first 8 weeks after implant placement, defect bridging in defects treated with

Table 2. Mechanical Properties of the Scaffolds Microarchitecture Types A-E: CoMponents Young's Modulus E (With Reference to The SMall $\varnothing 6$ Mm Cylinder), THE Yield STRENGTH $R_{0.2}$, AND THE Maximum Strength Before Structural Failure $\mathrm{R}_{\mathrm{M}}$

\begin{tabular}{lccc}
\hline Type & $\mathrm{E}\left(\mathrm{N} / \mathrm{mm}^{2}\right)$ & $\mathrm{R}_{0.2}\left(\mathrm{~N} / \mathrm{mm}^{2}\right)$ & $\mathrm{R}_{\mathrm{m}}\left(\mathrm{N} / \mathrm{mm}^{2}\right)$ \\
\hline $\mathrm{A}$ & $763 \pm 176$ & $12.1 \pm 0.4$ & $15.9 \pm 0.4$ \\
$\mathrm{~B}$ & $1804 \pm 400$ & $20.4 \pm 1.6$ & $38.5 \pm 1.1$ \\
$\mathrm{C}$ & $1870 \pm 250$ & $32.0 \pm 1.5$ & $98.0 \pm 11.1$ \\
$\mathrm{D}$ & $706 \pm 84$ & $16.8 \pm 2.5$ & $29.1 \pm 0.4$ \\
E & $1001 \pm 216$ & $19.3 \pm 1.5$ & $28.8 \pm 1.8$ \\
SLM Ti & $119 \mathrm{k} \pm 13.7 \mathrm{k}$ & $555 \pm 19.4$ & $662 \pm 20.6$ \\
\hline
\end{tabular}

SLM, selective laser melting. implants was almost complete (Fig. 5c). The BIC was in a very close range for all implant designs and not significantly different (Fig. 5d).

\section{Discussion}

Osteoconduction is a strong driving force in bone healing and particularly important when bone substitute materials are applied. In this study, we evaluated metallic bone substitutes with diverse microarchitectures. Such defined microarchitectures can be realized by the application of additive manufacturing; in this case, SLM of titanium powder. The results showed that the different microarchitectures with varying topological complexity had a substantial input on the mechanical properties but no input on the biological readout such as bone formation and bony bridging of the defects.

Histologically, there is no significant difference in bone ingrowth between the various microarchitectures. Despite the 5 open porous microarchitectures based on completely different architecture with different symmetries, accessibilities, connectivity, and anisotropies, their porosity $p$ is in a narrow range $72.3 \%<p<88.4 \%$ and very close to cancellous bone. ${ }^{20}$ Obviously, a minimal constriction of $290 \mu \mathrm{m}$ (type D) is enough for vascularization and tissue ingrowth, which is in agreement with ${ }^{13}$ stating that $150-500 \mu \mathrm{m}$ pores are optimal for porous biomaterial. In terms of minimal constriction, bone formation also occurs in pores of $50 \mu \mathrm{m}$ and below, ${ }^{21}$ but vascularization in such pores appears to be the limiting factor, since it was shown that bone formation in pores of $90-120 \mu \mathrm{m}$ induces osteochondral ossification, whereas those with larger diameters $(350 \mu \mathrm{m})$ induced bone formation directly within the tunnels. ${ }^{22}$ The maximal optimal pore size for Haversian-type bone formation was suggested to be $300-400 \mu \mathrm{m} .{ }^{22}$ A majority of articles on optimal maximal pore size suggest the largest evaluated pore size as optimal, which was in the range of $350-400 \mu \mathrm{m}^{23,24}$ In 2 articles, pores of $400-600 \mu \mathrm{m}$ performed worse than the ones between 350 and $400 \mu \mathrm{m}$ since pores in the range of $400-600 \mu \mathrm{m}$, occupied by adipocytes and bone marrow, lead to the formation of multiple capillaries ${ }^{25}$ and translated to a reduction of the mechanical properties. ${ }^{22}$ In a more recent study, ${ }^{14}$ cylindrical scaffolds of B-TCP were implanted in cancellous bone of sheep, but no difference in bone ingrowth was seen between pores in the range between 150 and $1220 \mu \mathrm{m}$, which is in line with our results. Due to the random distribution of these pores, the size of the constrictions, however, could not be determined.

In our scaffolds, constriction varied between $290 \mu \mathrm{m}$ (microarchitecture D) and $700 \mu \mathrm{m}$ (microarchitecture A) and pore size from $700 \mu \mathrm{m}$ (structure A) to $1300 \mu \mathrm{m}$ (microarchitecture C) and are thus more diverse and more defined than that of aforementioned scaffolds. Especially for larger scaffolds, the interconnection pathway could limit the accessibility and the vascularization. ${ }^{26}$ With the wide open porous structures from our studies, vascularization even with larger scaffolds should not be limiting, since the minimal constraint is $290 \mu \mathrm{m}$, which still allows Haversian-type bone formation that includes blood supply. ${ }^{22}$

An increase in surface between $1 \%$ and $42 \%$ had no effect on the biological readout. Since the surface roughness of all these scaffolds is the same, it suggests that the surface area in the range of the tested scaffolds has no effect on osteoconduction. 


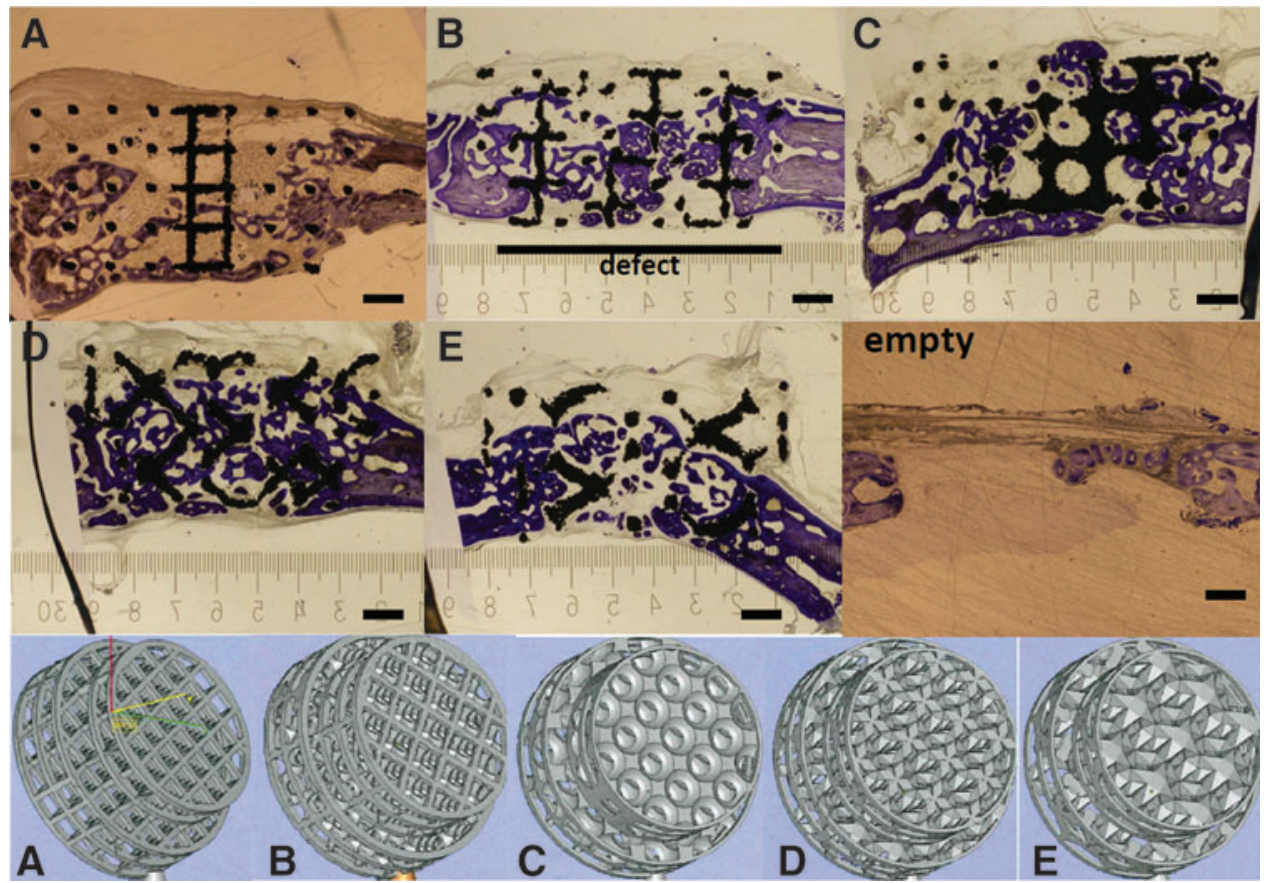

FIG. 4. Histological sections from the middle of the defect and illustrations of the implant designs $A-E$. For all scaffold designs from $A$ to $E$ and an empty defect, a histological section from one exemplary animal 8 weeks postoperatively is shown. Scale bars indicate $1 \mathrm{~mm}$. Original magnifications were 100-fold. Bone appears as grayish purple to purple. The size of the original defect is indicated in the histological section B.
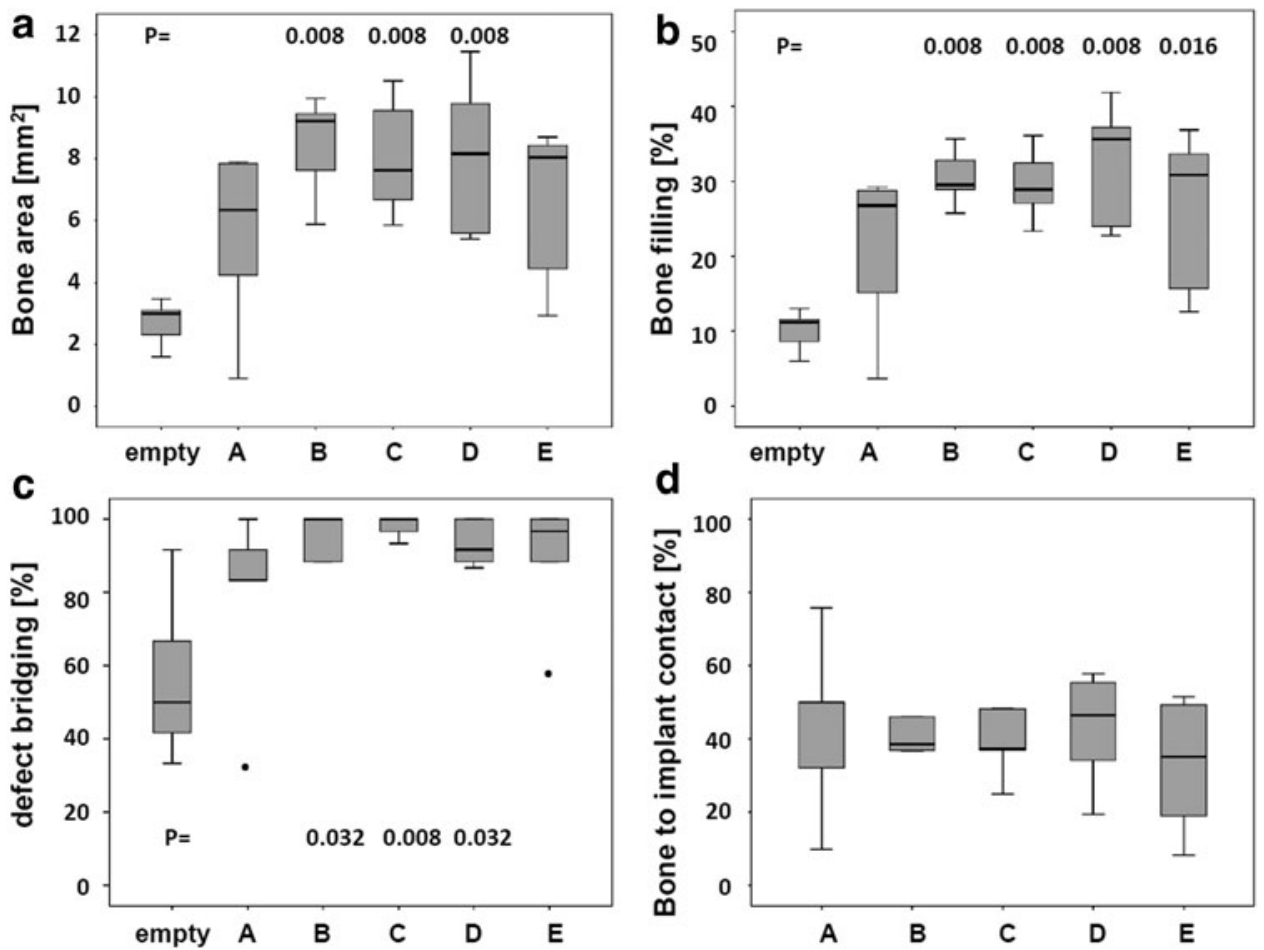

FIG. 5. Bone histomorphometric parameters in the area of interest (AOI). (a) The formation of new bone is significantly elevated compared to empty defects for the designs B, C, and D. (b) When taking into account that part of the AOI, occupied by titanium, the percentage of bone filling is significantly increased for type B, C, D, and E implants compared to the empty defect. (c) Defect bridging was significantly elevated compared to empty defects for the designs B, C, and D. (d) Between the different scaffold designs, no significant difference existed for bone to implant contact. Values are displayed as box plots ranging from the 25th (lower quartile) to the 75th (upper quartile) percentile, including the median as solid black line and whiskers, showing the minimum and maximum values. (c) Values outside the range of the box blot are shown as individual points. 
In a recent study, we showed that moderately rough surfaces as produced by sandblasting and acid etching had a significant effect on osteoconduction. ${ }^{15}$ A uniform surface treatment with sandblasting throughout large scaffolds can hardly be achieved or demand special architectures to allow the uniform penetration and impact of sand particles. ${ }^{27}$

Titanium scaffolds with concavities of $1600 \mu \mathrm{m}$ diameter and $800 \mu \mathrm{m}$ depth implanted in muscles induced bone formation preferentially in the concavities. ${ }^{28}$ Our scaffold (type C) resembles concavities of $1300 \mu \mathrm{m}$ diameter and $650 \mu \mathrm{m}$ depth, but showed no significant increase in bone formation compared to all other scaffolds, including microarchitectures based on squares (type A). The type A scaffold was also used in a former study to heal critical-sized defects in rabbits and proved to enhance defect bridging substantially to the same extent than in combination with osteoinductive growth factors or hydroxyapatite-based granules. ${ }^{29}$ Therefore, one can assume that round-shaped microarchitectures are not superior to rectangular-shaped ones in terms of osteoconduction. For osteoinduction, however, round shapes as in concavities appear to be more effective ${ }^{28,30}$ may be due to their resemblance to resorption lacunas generated by osteoclasts during bone degradation. ${ }^{31}$ Biomimetic osteoinductive surfaces were studied with sintered highly crystalline solid HA discs with hemispherical indentations of $2 \mathrm{~mm}$ as defined concavities on one or both planar surfaces. ${ }^{28,32}$ With microarchitecture $\mathrm{C}$, we realized pores of $1.36 \mathrm{~mm}$ in diameter throughout the scaffold but saw no indications for osteoinduction in terms of enhanced bone formation. For future studies, additive manufacturing will facilitate the realization of new designs to evaluate the effect of concavities and osteoinductive 3D designs in greater detail.

\section{Conclusion}

In future, additive manufacturing will enable the production of personalized scaffolds to treat patient's large bone defects more successfully. At present, it allows the realization of experimental scaffolds with an unlimited variety of microarchitectures to study the relationship between microarchitecture and enhanced bone regeneration by optimized osteoconduction. In this study, we show that osteoconduction of open porous bone substitutes with constrictions between 290 and $700 \mu \mathrm{m}$ and pore sizes between 800 and $1300 \mu \mathrm{m}$ is independent of the microarchitecture. The choice of the microarchitecture, however, has a great impact on the mechanical stability of the scaffold. Further studies are ongoing to elucidate the optimal microarchitecture for osteoconduction. Such an optimum exists since the empty defect with a constriction and pore size of $6000 \mu \mathrm{m}$ performs significantly worse than any microarchitecture tested in this study.

\section{Acknowledgment}

We thank Flora Nicholls, Ana Perez, and Alexander Tchouboukov for excellent technical assistance and Alexander Zwahlen for the 3D analysis of the structures. This research work was supported by a grant from the Swiss National Science Foundation (31003A_140868) and by the AOCMF (project C-10-37W), the Craniomaxillofacial Specialty of the AO Foundation.

\section{Author Contributions}

M.d.W.: Study design, surface characterization, main article writing. S.Z.: Design of the scaffolds. J.R.: Mechanical analysis. R.S.: Production of the scaffolds. T.F.: Surgical procedure, histology. C.G.: Surgical procedure, histomorphometry. F.E.W.: Study design, surgical procedure, data analysis, main article writing.

\section{Author Disclosure Statement}

No competing financial interests exist.

\section{References}

1. Sailer HF, Weber FE. [Bone substitutes]. Mund Kiefer Gesichtschir 2000;4 Suppl 1:S384-S391.

2. Brennan M, Renaud A, Amiaud J, et al. Pre-clinical studies of bone regeneration with human bone marrow stromal cells and biphasic calcium phosphate. Stem Cell Res Ther 2014; 5:114.

3. Cancedda R, Dozin B, Giannoni P, et al. Tissue engineering and cell therapy of cartilage and bone. Matrix Biol 2003; 22:81-91.

4. Urist MR. Bone: Formation by autoinduction. Science 1965; 150:893-899.

5. Martin BI, Lurie JD, Tosteson ANA, et al. Use of bone morphogenetic protein among patients undergoing fusion for degenerative diagnoses in the United States, 2002 to 2012. Spine J 2015;15:692-699.

6. Barth A. Ueber histologische Befunde nach Knochenimplantationen. Langenbecks Arch klin Chir 1893;46:409. [Sonder-Abdruck aus v. Langenbeck's Archiv, Bd. XLVI, Heft 2] In German.

7. Albrektsson T, Johansson C. Osteoinduction, osteoconduction and osseointegration. Eur Spine J 2001;10:S96-S101.

8. Chia H, Wu B. Recent advances in 3D printing of biomaterials. J Biol Eng 2015;9:4.

9. Le Guehennec L, Soueidan A, Layrolle P, et al. Surface treatments of titanium dental implants for rapid osseointegration. Dent Mater 2007;23:844-854.

10. Imwinkelried T. Mechanical properties of open-pore titanium foam. J Biomed Mater Res A 2007;81:964-970.

11. Levine BR, Sporer S, Poggie RA, et al. Experimental and clinical performance of porous tantalum in orthopedic surgery. Biomaterials 2006;27:4671-4681.

12. Bansiddhi A, Sargeant TD, Stupp SI, et al. Porous NiTi for bone implants: A review. Acta Biomater 2008;4: 773-782.

13. Jones AC, Arns CH, Hutmacher DW, et al. The correlation of pore morphology, interconnectivity and physical properties of 3D ceramic scaffolds with bone ingrowth. Biomaterials 2009; 30:1440-1451.

14. von Doernberg M-C, von Rechenberg B, Bohner M, et al. In vivo behavior of calcium phosphate scaffolds with four different pore sizes. Biomaterials 2006;27: 5186-5198.

15. de Wild M, Schumacher R, Mayer K, et al. Bone regeneration by the osteoconductivity of porous titanium implants manufactured by selective laser melting: A histological and micro computed tomography study in the rabbit. Tissue Eng Part A 2013;19:2645-2654.

16. Skyscan Morphometric parameters measured by Skyscan ${ }^{\mathrm{TM}}$ CT-analyser software, Skyscan N.V, 2007. Available at: www.bruker-microct.com/next/CTAn03.pdf (accessed August 22, 2016). 
17. ASTM Standard Specification for Unalloyed Titanium, for Surgical Implant Applications (UNS R50250, UNS R50400, UNS R50550, UNS R50700) American Society for Testing and Measurement, 2003.

18. Kruse A, Jung RE, Nichols F, et al. Bone regeneration in the presence of a synthetic hydroxyapatite oxide based and a xenogenic hydroxyapatite based bone substitute material. Clin Oral Implants Res 2011;22:506-511.

19. Schmidlin PR, Tchouboukov A, Wegehaupt FJ, et al. Effect of cerium chloride application on fibroblast and osteoblast proliferation and differentiation. Arch Oral Biol 2012;57: 892-897.

20. Wagoner Johnson AJ, Herschler BA. A review of the mechanical behavior of $\mathrm{CaP}$ and $\mathrm{CaP} /$ polymer composites for applications in bone replacement and repair. Acta Biomater 2011;7:16-30.

21. Itala AI, Ylanen HO, Ekholm C, et al. Pore diameter of more than 100 microm is not requisite for bone ingrowth in rabbits. J Biomed Mater Res 2001;58:679-683.

22. Kuboki Y, Jin Q, Takita H. Geometry of carriers controlling phenotypic expression in BMP-induced osteogenesis and chondrogenesis. J Bone Joint Surg 2001;83:S105-S115.

23. Galois L, Mainard D. Bone ingrowth into two porous ceramics with different pore sizes: An experimental study. Acta Orthop Belg 2004;70:598-603.

24. Murphy CM, Haugh MG, O'Brien FJ. The effect of mean pore size on cell attachment, proliferation and migration in collagen-glycosaminoglycan scaffolds for bone tissue engineering. Biomaterials 2010;31:461-466.

25. Tsuruga E, Takita $\mathrm{H}$, Itoh $\mathrm{H}$, et al. Pore Size of porous hydroxyapatite as the cell-substratum controls BMPinduced osteogenesis. J Biochem 1997;121:317-324.

26. Mastrogiacomo M, Scaglione S, Martinetti R, et al. Role of scaffold internal structure on in vivo bone formation in macroporous calcium phosphate bioceramics. Biomaterials 2006;27:3230-3237.

27. Butscher A, Bohner M, Doebelin N, et al. New depowderingfriendly designs for three-dimensional printing of calcium phosphate bone substitutes. Acta Biomater 2013;9:9149_ 9158.

28. Ripamonti U, Roden LC, Renton LF. Osteoinductive hydroxyapatite-coated titanium implants. Biomaterials 2012; 33:3813-3823.

29. Karfeld-Sulzer LS, Ghayor C, Siegenthaler B, et al. Nmethyl pyrrolidone/bone morphogenetic protein-2 double delivery with in situ forming implants. J Control Release 2015;203:181-188.

30. Rumpler M, Woesz A, Varga F, et al. Three-dimensional growth behavior of osteoblasts on biomimetic hydroxylapatite scaffolds. J Biomed Mater Res A 2007;81:40-50.

31. Robling AG, Castillo AB, Turner $\mathrm{CH}$. Biomechanical and molecular regulation of bone remodeling. Annu Rev Biomed Eng 2006;8:455-498.

32. Ripamonti U, Richter PW, Nilen RWN, et al. The induction of bone formation by smart biphasic hydroxyapatite tricalcium phosphate biomimetic matrices in the non-human primate Papio ursinus. J Cell Mol Med 2008;12:2609-2621.

Address correspondence to:

Franz E. Weber

Oral Biotechnology \& Bioengineering

Center of Dental Medicine

University of Zurich

Plattenstrasse 11

Zurich 8032

Switzerland

E-mail: franz.weber@zzm.uzh.ch 